\title{
Erratum to: Engineering redox cofactor utilization for detoxification of glycolaldehyde, a key inhibitor of bioethanol production, in yeast Saccharomyces cerevisiae
}

Lahiru N. Jayakody • Kenta Horie • Nobuyuki Hayashi • Hiroshi Kitagaki

Published online: 21 June 2014

(C) Springer-Verlag Berlin Heidelberg 2014

Erratum to: Appl Microbiol Biotechnol (2013) 97:6589-6600

DOI 10.1007/s00253-013-4997-4

The original version of this article inadvertently contained mistake in Table 2.

Table 2

Wrong (present)

SalI-CCC GAC CTA CCA TTT TGT GAA TCA A

Correct

SalI - CCC GTC GAC CTA CCA TTT TGT GAA TCA A

The online version of the original article can be found at http://dx.doi.org/ 10.1007/s00253-013-4997-4.

L. N. Jayakody $\cdot$ K. Horie $\cdot$ N. Hayashi $\cdot$ H. Kitagaki $(\bowtie)$

Department of Environmental Science, Faculty of Agriculture, Saga

University, Saga 8408502, Japan

e-mail: ktgkhrs@cc.saga-u.ac.jp

L. N. Jayakody $\cdot$ N. Hayashi $\cdot$ H. Kitagaki

Department of Biochemistry and Applied Biosciences, United

Graduate School of Agricultural Sciences Kagoshima, Kagoshima

University, Korimoto 1-21-24, Kagoshima 890-8580, Japan 